\title{
Macrodissection prior to closed system RT-qPCR is not necessary for estrogen receptor and HER2 concordance with IHC/FISH in breast cancer
}

\author{
Swati Gupta ${ }^{1} \cdot$ Navin R. Mani ${ }^{1}$ - Daniel E. Carvajal-Hausdorf ${ }^{1,2} \cdot$ Veerle Bossuyt $^{1} \cdot$ Kenneth Ho $^{3} \cdot$ Jodi Weidler $^{4}$. \\ Wendy Wong ${ }^{3} \cdot$ Brian Rhees $^{3} \cdot$ Michael Bates $^{4} \cdot$ David L. Rimm $^{1}$
}

Received: 10 December 2017 / Revised: 7 February 2018 / Accepted: 28 February 2018 / Published online: 1 June 2018

(c) United States \& Canadian Academy of Pathology 2018

\begin{abstract}
An on-demand, closed RT-qPCR, the GeneXpert (GX) system, has the potential to provide biomarker information in lowresourced settings and elsewhere. We used this system with a research use only version of the Breast Cancer STRAT4 cartridge that measures the mRNA expression levels of ERBB2, ESR1, PGR, and MKi67. Here we evaluated the impact of non-macrodissected (non $\mathrm{m}-\mathrm{d}$ ) versus macrodissected $(\mathrm{m}-\mathrm{d})$ samples using STRAT4 on formalin-fixed, paraffin-embedded (FFPE) core needle biopsies. Two cohorts were assessed: (1) 60 FFPE infiltrating ductal carcinoma (IDCA) cases and (2) 20 FFPE IDCA cases with ductal carcinoma in situ (DCIS) with a range of HER2 expression as determined by clinical immunohistochemistry and fluorescence in situ hybridization (IHC/FISH). We observed about half of the core needle biopsy area as invasive tumor in both IDCA (mean $=51.5 \%$ ) and IDCA with DCIS (mean $=53.5 \%$ ) cohorts, but also found the mRNA levels were independent of tumor area. We found excellent agreement of the mRNA transcript level between the paired samples, m-d versus non m-d, for ERBB2, ESR1, PGR, and MKi67 for both the IDCA and IDCA with DCIS cohorts. No significant difference $(P>0.99)$ was observed when we compared the mRNA transcript level between the paired samples $\mathrm{m}$-d versus non $\mathrm{m}$-d. In addition, we noted a significant concordance $(P<0.001)$ between RT-qPCR and IHC/FISH for HER2-positivity, ER-positivity, and PR-positivity, independent of specimen dissection. These data suggest that mRNA expression for $E R B B 2, E S R$, and $P G R$ is sufficiently low in surrounding tissue cells such that macrodissection is not required for assessment of key breast cancer mRNA markers and is independent of the amount of input tumor. This approach may be valuable in settings lacking pathology expertise or using specimen types, such as fine-needle aspirates, where it may be challenging to separate non-tumor from tumor tissue.
\end{abstract}

Electronic supplementary material The online version of this article (https://doi.org/10.1038/s41374-018-0064-1) contains supplementary material, which is available to authorized users.

David L. Rimm

david.rimm@yale.edu

1 Department of Pathology, Yale University School of Medicine, New Haven, CT, USA

2 Anatomía Patológica, Clínica Alemana-Facultad de Medicina Universidad del Desarrollo, Santiago, Chile

3 Division of Oncology Research and Development, Oncology, Cepheid, Sunnyvale, CA, USA

4 Medical and Scientific Affairs and Strategy, Oncology, Cepheid, Sunnyvale, CA, USA

\section{Introduction}

Breast cancer is one of the most common cancers in women worldwide in both developing and developed countries [1,2]. Worldwide, newly diagnosed breast cancer cases are estimated at $\sim 1.7$ million annually, with the highest numbers observed in Europe (30\%) and the US (12\%) [2]. Over the years, breast cancer mortality in the developed world has decreased by $36 \%$ due to improved treatment and early detection [3]. Overexpression of human epidermal growth factor (HER2/ERBB2) at the gene and protein level is a standard of care in the US and Europe for breast cancer patients since it is predictive of response to HER2-targeted therapies $[4,5]$. Estrogen receptor $(\mathrm{ESR} / E S R I)$ and progesterone receptor $(\mathrm{PGR} / P G R)$ are prognostic factors for aggressive disease and predict response to tamoxifen and other hormonal therapies [6-9]. 

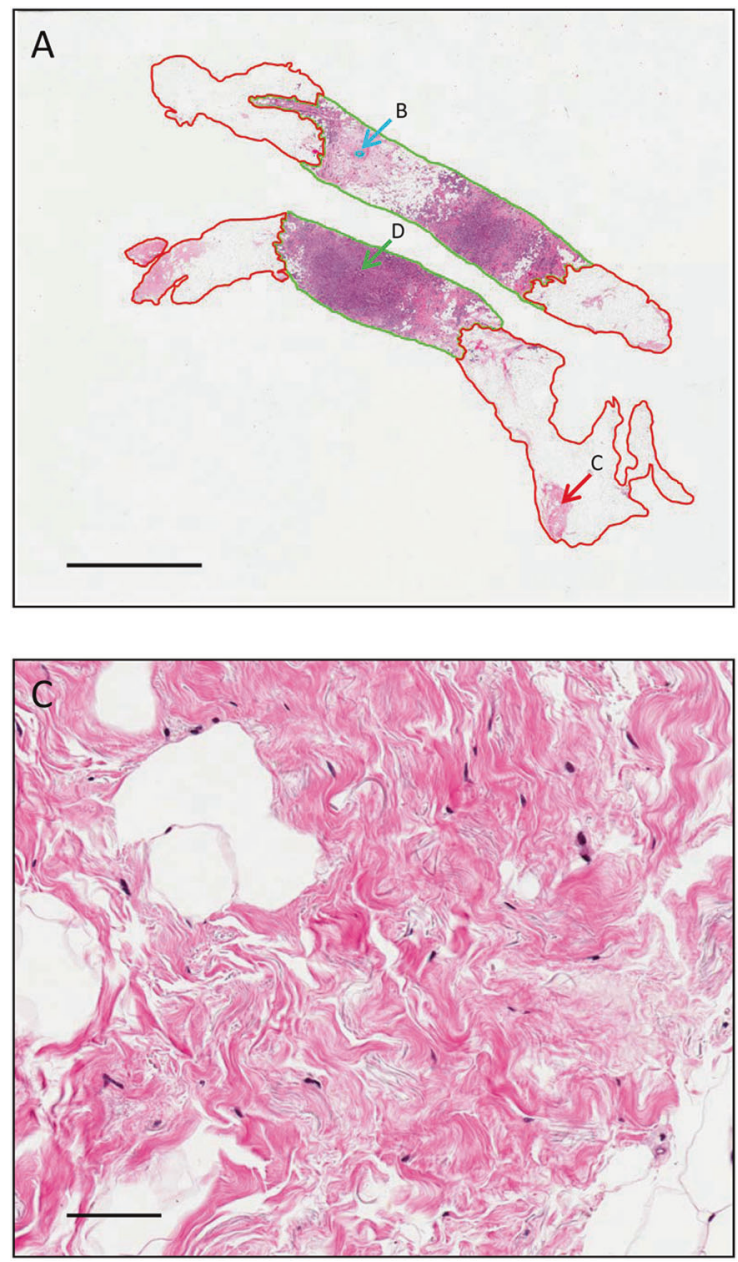

Fig. 1 Representative images of H\&E stained IDCA with DCIS breast cancer section showing overall view of the core needle biopsy (a), DCIS tumor area (b), non-tumor tissue area (c), and tumor tissue area

The measurement of HER2, ER, PR, and sometimes Ki-67 protein expression by immunohistochemistry (IHC) represents the standard of care in the clinic. In addition, ERBB2 copy number status is often assessed by fluorescence in situ hybridization (FISH) when HER2 IHC results are equivocal [10]. While mRNA is used to assess these same markers as part of the commonly used Oncotype Dx test, mRNA is not commonly used as the basis for therapeutic regimen selection, and is not endorsed in the ASCO/CAP guidelines [11]. One reason for this is that the in situ value of IHC tests is thought to prevent the false positive tests that might be seen due to contaminating mRNA expression for these key markers in nontumor tissue.

Here we determine whether mRNA expression for key breast cancer markers, ERBB2, ESR1, PGR, and MKi67, is equivalent in non-macrodissected sections (non m-d) compared with tumor-specific macrodissected sections (m-d) using core needle biopsies with infiltrating ductal carcinoma (IDCA) and IDCA with ductal carcinoma in situ (DCIS) using a real-time quantitative reverse transcription polymerase chain reaction
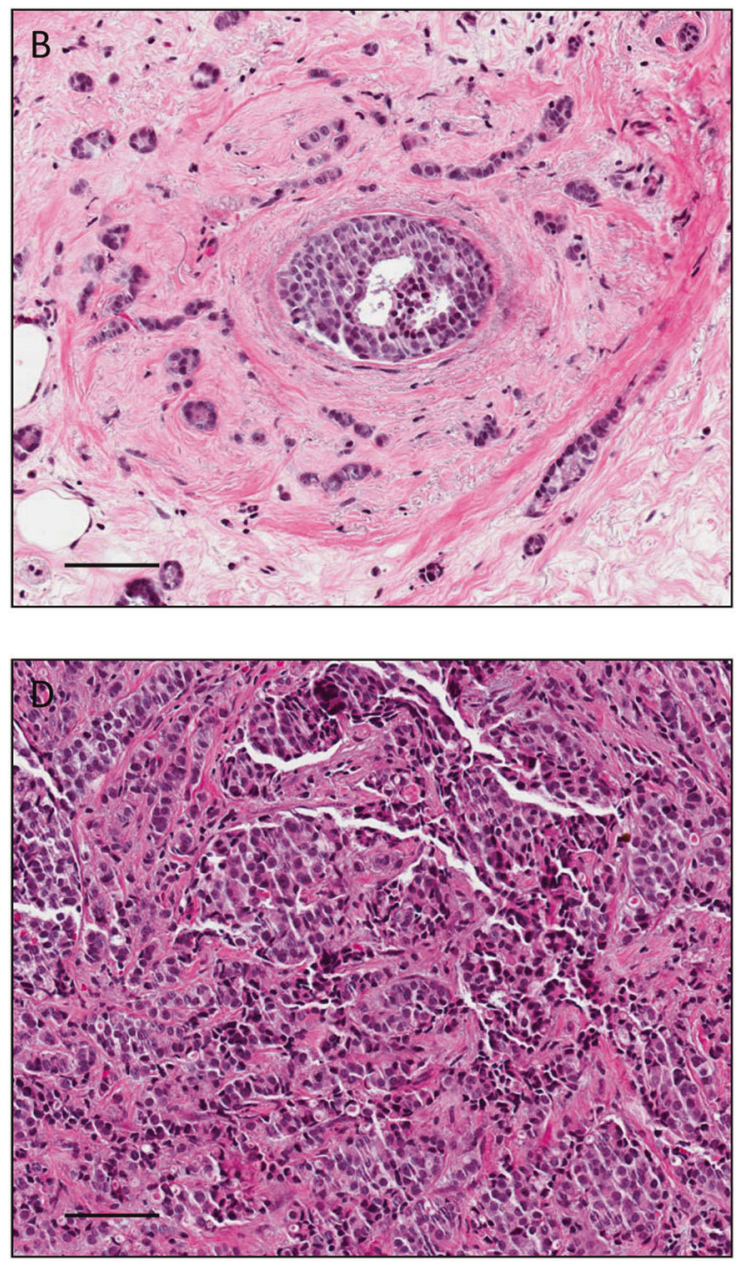

(d). Magnification panels and scale bar shown at (a) are $\times 0.6$ and 4 $\mathrm{mm}$ and $\mathbf{b}-\mathbf{d}$ are $\times 20$ and $100 \mathrm{um}$. Green represents tumor area, red represents stroma area, and cyan represents DCIS area

(RT-qPCR) assay (GeneXpert Breast Cancer STRAT4 RUO Assay, Cepheid, Sunnyvale, CA, USA). Additionally, we assessed the correlation between RT-qPCR and clinically determined IHC/FISH for HER2-positivity, ER-positivity, and PgR-positivity in the paired samples, non m-d versus m-d.

\section{Materials and methods}

\section{Patient and tissues}

Breast carcinoma patient cohorts were selected by screening the tumor bank of Yale University for 60 formalin-fixed, paraffin-embedded (FFPE) IDCA and 20 FFPE IDCA with DCIS diagnosed between 2012 and 2015. The results for CLIA-certified HER2 IHC/FISH scoring according to the American Society of Clinical Oncology, and the College of American Pathologists (ASCO/CAP) guidelines were extracted from the pathology reports. The tissues were collected under IRB protocol ID 9505008219. 
Table 1 Percentage epithelial tumor area in the IDCA cohort

$\%$ Tumor epithelium, HER2 $0+, n=13$

Mean

Median

SD

Minimum

Maximum

$\%$ Tumor epithelium, HER2 $1+, n=17$

Mean

Median

SD

Minimum

Maximum

$\%$ Tumor epithelium, HER2 $2+, n=16$

Mean

Median

SD

Minimum

Maximum

$\%$ Tumor epithelium, HER2 $3+, n=14$

Mean

Median

SD

Minimum

Maximum

\section{Area measurement}

Samples were sectioned at the Yale pathology tissue service. Imaging of hematoxylin and eosin sections was completed on Aperio ScanScope Console (v10.2.0.2352). Tumor, stroma, and DCIS areas circled by a pathologist were measured using pen and annotation tool of Aperio ImageScope (v12.3.2.8013).

\section{GeneXpert Breast Cancer STRAT4 assay}

The BC STRAT4 assay was performed as previously described [12]. Briefly, 5- $\mu \mathrm{M}$-thick FFPE tissue sections were collected either as a whole tissue section, non m-d, or macrodissected, m-d, to collect tumor only. Samples were lysed using $5 \mu \mathrm{l}$ Proteinase $\mathrm{K}$ and $260 \mu \mathrm{l}$ FFPE lysis reagent and incubated for $30 \mathrm{~min}$ at $80{ }^{\circ} \mathrm{C}$. Then, $260 \mu \mathrm{L}$ of $\geq 95 \%$ ethanol was added to the lysed samples and vortexed to mix. The mixture was transferred to a BC STRAT4 cartridge and tested on the GeneXpert (GX) system. This assay isolates the total RNA, performs a 1-step RT-PCR and provides $\mathrm{Ct}$ values for the endogenous control, CYFIP, and the target genes, ERBB2, ESR1, PGR, and MKi67. Results were expressed as a $\mathrm{dCt}$ value, defined as the $\mathrm{Ct}$ of the control gene (CYFIP1) minus the $\mathrm{Ct}$ of each of the target
Table 2 Percentage epithelial tumor and DCIS area in the IDCA with DCIS cohort

\begin{tabular}{lll}
\hline & $\%$ Tumor epithelium & $\%$ DCIS \\
\hline HER2 $0+, n=5$ & & \\
Mean & 46.8 & 0.6 \\
Median & 28 & 0.4 \\
SD & 29.9 & 0.6 \\
Minimum & 22 & 0.1 \\
Maximum & 81 & 1.5 \\
HER2 1+, $n=8$ & & \\
Mean & 55.3 & 1.2 \\
Median & 55.5 & 0.7 \\
SD & 18.1 & 1.2 \\
Minimum & 20 & 0.1 \\
Maximum & 77 & 3.9 \\
HER2 2+, $n=7$ & & \\
Mean & 58.6 & 1.7 \\
Median & 52 & 0.9 \\
SD & 24.5 & 2.0 \\
Minimum & 30 & 0.1 \\
Maximum & 97 & 5.4 \\
\hline
\end{tabular}

genes (ERBB2, ESR1, PGR, or MKi67). The BC STRAT4 $\mathrm{dCt}$ cutoffs for ERBB2, ESR, PGR, and MKi67 were established in a breast cancer patient cohort, based on the highest concordance achieved between relative RNA amplification and corresponding protein expression by IHC as the gold standard [13].

\section{Statistical analysis}

All datasets were analyzed and plotted using GraphPad Prism v7.0 software for Windows (GraphPad Software, Inc., La Jolla, CA). The concordance between non m-d and $\mathrm{m}$-d groups was assessed using linear regression coefficient $\left(R^{2}\right)$. The comparison amongst non $\mathrm{m}-\mathrm{d}$ and $\mathrm{m}$-d groups was performed using two-tailed unpaired Student's $t$-tests. Fisher's exact test two-sided was used to compare the RTqPCR and IHC results for HER2-positivity, ER-positivity, and PgR-positivity. All $P$-values under 0.05 were considered statistically significant.

\section{Results}

\section{Tumor epithelium and DCIS area in cohorts}

Tumor epithelium and DCIS were circled and the percentage of tumor area was measured to assess the stroma and DCIS components present in all breast cancer samples (Fig. 1). The distribution of percentage tumor epithelium 
Fig. 2 Correlation between non $\mathrm{m}$-d versus $\mathrm{m}$-d samples for $\mathrm{RT}$ qPCR in IDCA cohort. Regression of mRNA quantification for $E R B B 2(\mathbf{a})$, ESR1 (b), PGR (c), and MKi67 (d). The respective $R^{2}$ and slope values are indicated in the chart. Non m-d not macrodissected, md macrodissected

Fig. 3 Correlation between non $m-d$ versus $m-d$ samples for RTqPCR in IDCA with DCIS cohort. Regression of mRNA quantification for ERBB2 (a), ESR1 (b), PGR (c), and MKi67 (d). The respective $R^{2}$ and slope values are indicated in the chart. Non m-d not macrodissected, m$\mathrm{d}$ macrodissected
A

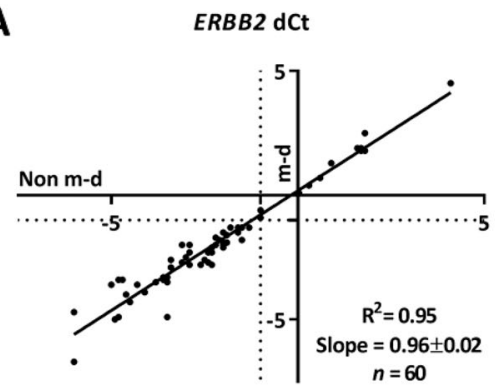

C

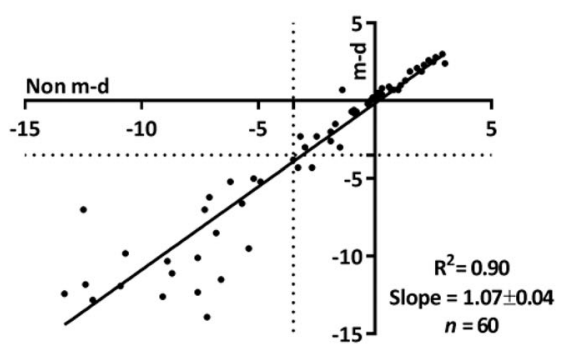

A

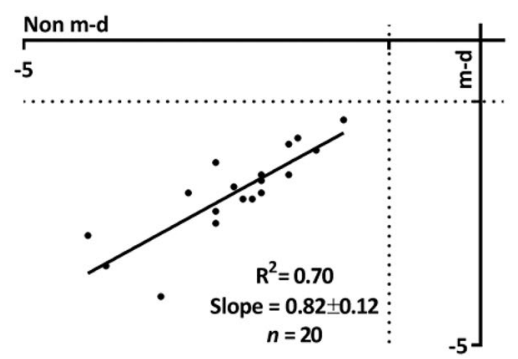

C

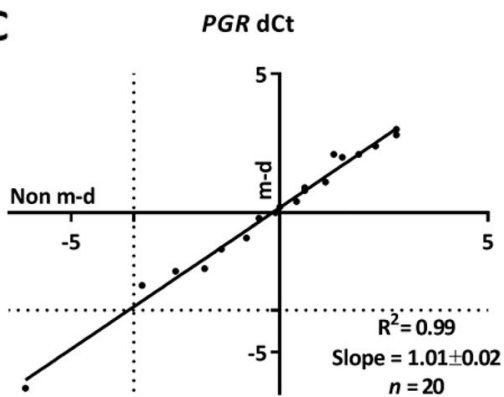

B

ESR1 dCt

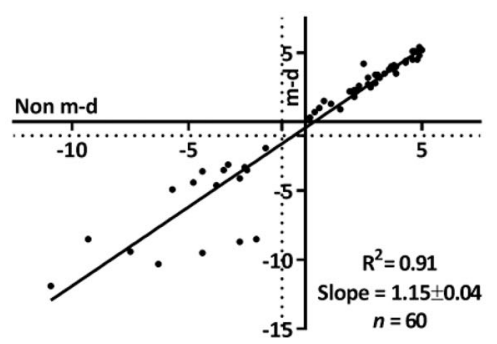

D

MKi67 dCt

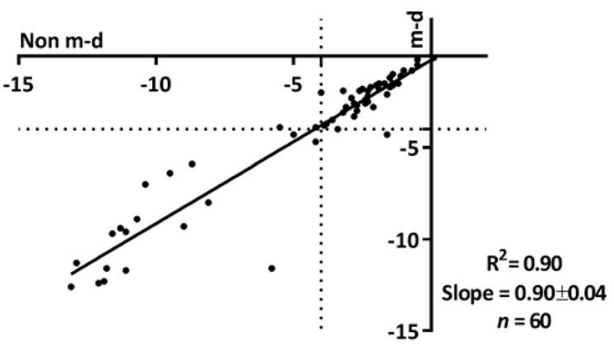

B

ESR1 dCt

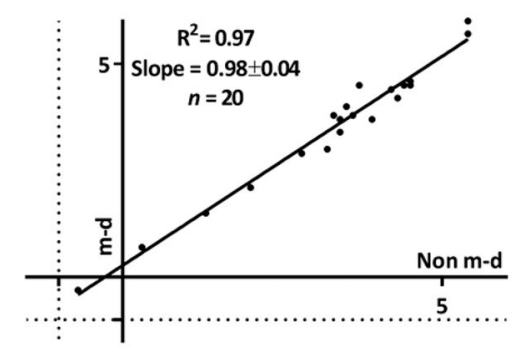

D

MKi67 dCt

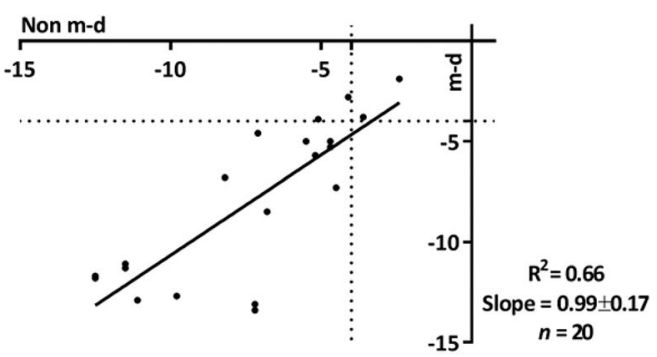

area in the IDCA cohort are shown in Table 1. Similarly, the distributions of percentage tumor epithelium and percentage DCIS area in the IDCA cohort is shown in Table 2. We observed about half of the core needle biopsy area as stroma in both the IDCA (mean percentage tumor $=51.5 \%$ ) and IDCA with DCIS (mean percentage tumor $=53.5 \%$ ) cohorts. The percentage tumor epithelium in this small cohort ranges from 13 to $100 \%$. In addition, we found a range of DCIS positivity of $0.1-5.4 \%$ in the IDCA with DCIS cohort.

\section{Breast cancer mRNA markers assessed as a function of macrodissection}

To evaluate the impact of macrodissection, we measured mRNA expression of key breast cancer genes, ERBB2, ESR1, PGR, and MKi67, between the paired samples non $\mathrm{m}-\mathrm{d}$ versus $\mathrm{m}$-d from 60 FFPE IDCA cases and 20 FFPE IDCA with DCIS cases with a range of HER2 expression determined by HER2 IHC/FISH. We saw excellent agreement of the resulting $\mathrm{dCt}$ values, between the paired 
Table 3 Concordance between RT-qPCR and IHC/FISH for HER2 in IDCA cohort

\begin{tabular}{lllc}
\hline & HER2 positive & HER2 negative & Total \\
\hline \multicolumn{2}{l}{ Non m-d samples $(P<0.001$; sensitivity } & $=0.86$; specificity $=1 ;$ PPV \\
$=1 ; \mathrm{NPV}=0.96)$ & & 12 \\
$\mathrm{dCt} \geq-1$ & 12 & 0 & 48 \\
$\mathrm{dCt}<-1$ & 2 & 46 & 60 \\
Total & 14 & 46 & \\
m-d samples $(P<0.001 ;$ sensitivity $=0.86 ;$ specificity $=1 ;$ PPV $=1 ;$ \\
NPV $=0.96)$ & & 0 & 12 \\
$\mathrm{dCt} \geq-1$ & 12 & 46 & 48 \\
$\mathrm{dCt}<-1$ & 2 & 46 & 60 \\
Total & 14 &
\end{tabular}

Table 4 Concordance between RT-qPCR and IHC/FISH for ESR in IDCA cohort

\begin{tabular}{|c|c|c|c|}
\hline & ER positive & ER negative & Total \\
\hline \multicolumn{4}{|c|}{$\begin{array}{l}\text { Non } \mathrm{m} \text {-d samples }(P<0.001 \text {; sensitivity }=0.98 ; \text { specificity }=1 ; \mathrm{PPV} \\
=1 ; \mathrm{NPV}=0.94)\end{array}$} \\
\hline $\mathrm{dCt} \geq-1$ & 43 & 0 & 43 \\
\hline $\mathrm{dCt}<-1$ & 1 & 16 & 17 \\
\hline Total & 44 & 16 & 60 \\
\hline \multicolumn{4}{|c|}{$\begin{array}{l}\mathrm{m}-\mathrm{d} \text { samples }(P<0.001 ; \text { sensitivity }=0.98 ; \text { specificity }=1 ; \mathrm{PPV}=1 \text {; } \\
\mathrm{NPV}=0.94)\end{array}$} \\
\hline $\mathrm{dC} t \geq-1$ & 43 & 0 & 43 \\
\hline $\mathrm{dCt}<-1$ & 1 & 16 & 17 \\
\hline Total & 44 & 16 & 60 \\
\hline
\end{tabular}

Fig. 4 Comparison between pathologists determined $\mathrm{IHC} /$ FISH and RT-qPCR for non m-d versus $m-d$ samples in IDCA cohort. Dot plot showing mRNA quantification of ERBB2 (a), ESR1 (b), PGR (c), and MKi67 (d) determined to be $0+, 1+$, $2+$, and $3+$ by IHC and confirmed by FISH. Non m-d not macrodissected, m-d: macrodissected
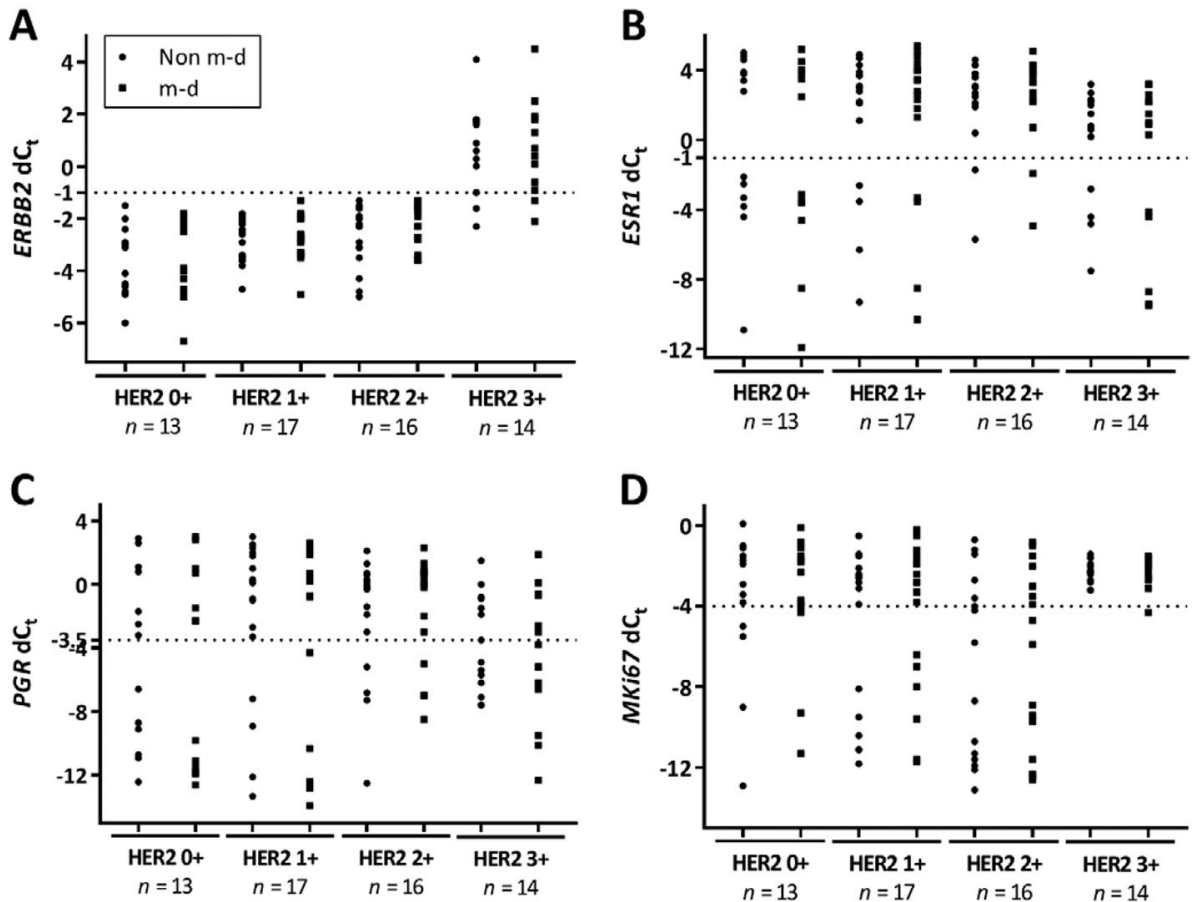

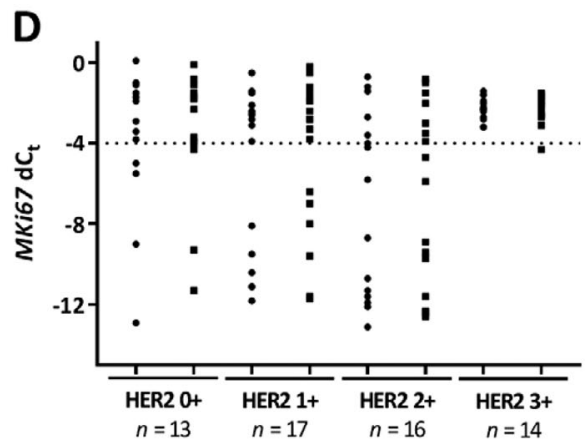

samples, m-d versus non m-d, for ERBB2 $\left(R^{2}=0.95\right), E S R 1$ $\left(R^{2}=0.91\right), P G R\left(R^{2}=0.90\right)$, and $\operatorname{MKi67}\left(R^{2}=0.90\right)$ in the IDCA cohort (Fig. 2). Since contaminating DCIS in IDCA specimens could potentially contribute ERBB2 mRNA signal to in vitro assays, a second cohort was assessed where DCIS accompanied IDCA in every case. Here high concordance of the resulting $\mathrm{dCt}$ between the paired samples, m-d versus non m-d, was observed for ERBB2 $\left(R^{2}=0.70\right)$, ESRl $\left(R^{2}=0.97\right), P G R\left(R^{2}=0.99\right)$, and $\operatorname{MKi67}\left(R^{2}=0.66\right)$ (Fig. 3). Experiments were performed in triplicates for cases closer to the $E R B B 2$ threshold $(-0.5<\mathrm{dCt}<-1.5)$ and the agreement between non $\mathrm{m}$-d versus $\mathrm{m}$-d samples for every case was observed (Supplemental Fig. 1). When clinical cutoffs for the various analytes are applied, the concordance remains strong, no specimen in either cohort changed from positive to negative or vice versa as a function of non $\mathrm{m}$-d or m-d for ERBB2 and ESR (Tables 3 and 4). In addition, no significant difference $(P>0.99)$ was observed when we compared the $\mathrm{dCt}$ between the paired samples, m-d versus non $\mathrm{m}-\mathrm{d}$, for $E R B B 2, E S R 1, P G R$, and $M K i 67$ in the IDCA cohort (Fig. 4) and IDCA with DCIS cohort (Fig. 5).

\section{Concordance between RT-qPCR and IHC/FISH measurements}

To assess the concordance agreement between RT-qPCR and IHC/FISH classification, we used the predefined BC STRAT4 dCt cutoffs for ERBB2, ESR, and PGR positivity and compared those to clinical IHC/FISH results in the IDCA cohort $[12,13]$. We found a significant concordance 
Fig. 5 Comparison between pathologist determined IHC/ FISH and RT-qPCR for non m-d versus $\mathrm{m}$-d samples in IDCA with DCIS cohort. Dot plot showing mRNA quantification of ERBB2 (a), ESR1 (b), PGR (c), and MKi67 (d) determined to be $0+, 1+$, and $2+$ by IHC and confirmed by FISH. Non $\mathrm{m}$ $\mathrm{d}$ not macrodissected, $\mathrm{m}-\mathrm{d}$ macrodissected
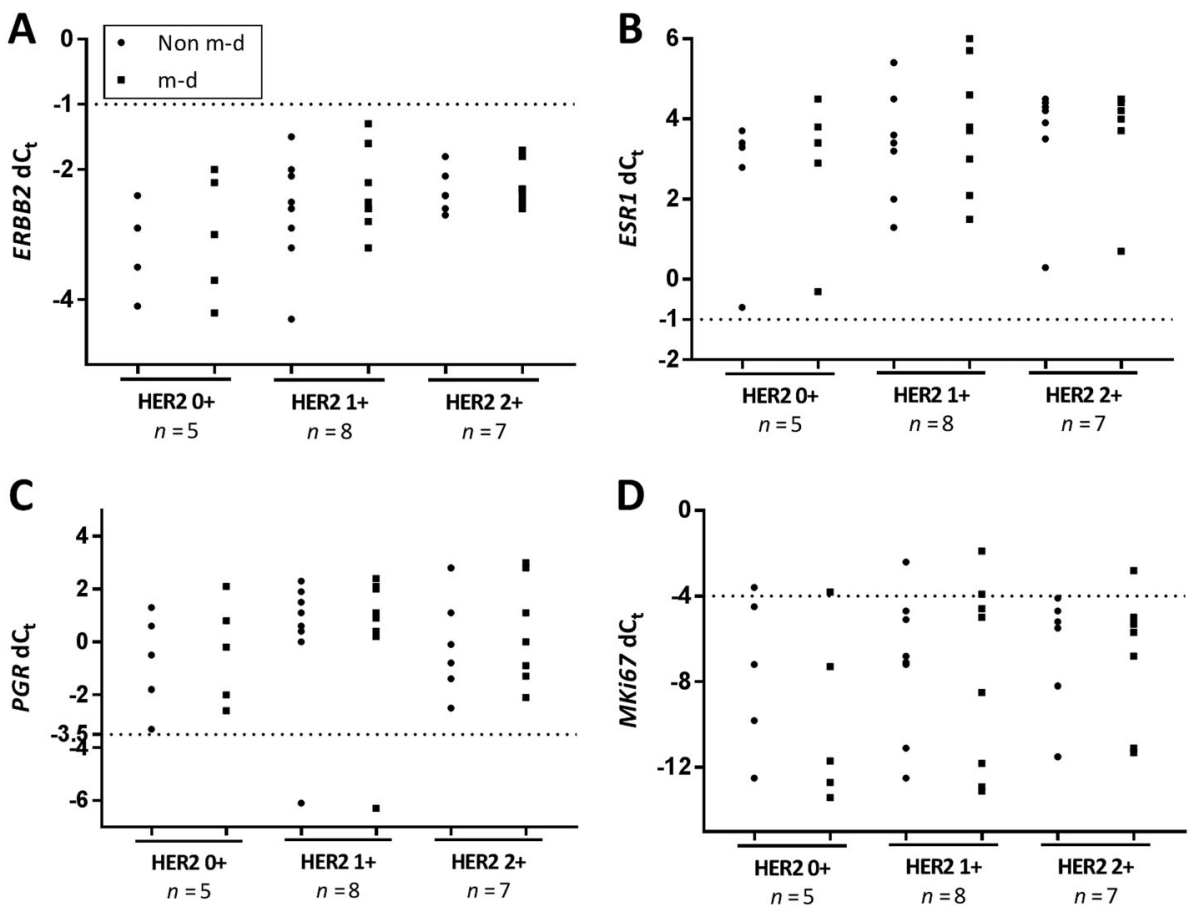

Table 5 Concordance between RT-qPCR and IHC/FISH for PGR in IDCA cohort

PR positive PR negative Total

Non $\mathrm{m}$-d samples $(P<0.001$; sensitivity $=0.86$; specificity $=0.88$; $\mathrm{PPV}=0.95 ; \mathrm{NPV}=0.71)$

$\begin{array}{llll}\mathrm{dCt} \geq-3.5 & 37 & 2 & 39 \\ \mathrm{dCt}<-3.5 & 6 & 15 & 21 \\ \text { Total } & 43 & 17 & 60\end{array}$

m-d samples $(P<0.001$; sensitivity $=0.84 ;$ specificity $=1 ; \mathrm{PPV}=1$; $\mathrm{NPV}=0.71)$

\begin{tabular}{llll}
$\mathrm{dCt} \geq-3.5$ & 36 & 0 & 36 \\
$\mathrm{dCt}<-3.5$ & 7 & 17 & 24 \\
Total & 43 & 17 & 60 \\
\hline
\end{tabular}

between RT-qPCR and IHC/FISH for HER2-positivity for the paired samples, non m-d and m-d $(95.0 \% ; P<0.001$; sensitivity $=0.86 ; \quad$ specificity $=1 ; \quad \mathrm{PPV}=1 ; \quad \mathrm{NPV}=0.96$ ) (Table 3), ESR-positivity for the paired samples, non m-d and m-d $(98.3 \% ; P<0.001 ;$ sensitivity $=0.98 ;$ specificity $=1$; $\mathrm{PPV}=1 ; \mathrm{NPV}=0.94)$ (Table 4) and PGR-positivity for the paired samples, non $\mathrm{m}-\mathrm{d}(86.7 \% ; P<0.001$; sensitivity $=$ 0.86 ; specificity $=0.88 ; \mathrm{PPV}=1 ; \mathrm{NPV}=0.71$ ) versus $\mathrm{m}-\mathrm{d}$ $(88.3 \% ; P<0.001 ;$ sensitivity $=0.84$; specificity $=1 ; \mathrm{PPV}=$ $1 ; \mathrm{NPV}=0.71)($ Table 5$)$, respectively.

\section{RT-qPCR dCt scores were independent of tumor epithelium and DCIS area}

We assessed the area-specific distribution of mRNA expression in both the IDCA and IDCA with DCIS cohorts.
The mRNA transcript levels of ERBB2, ESR1, PGR, and $M K i 67$ were independent of the percent epithelial tumor area present in the IDCA and IDCA with DCIS cohorts (Fig. 6a, b and data not shown). Furthermore, the mRNA transcript levels of ERBB2, ESR1, PGR, and MKi67 were also independent of the percent DCIS area present in IDCA with DCIS cohort (Fig. 6c, d and data not shown).

\section{Discussion}

Breast cancer tissues show both architectural and cellular heterogeneity, representing a blend of tumor cells, healthy epithelial ducts, surrounding stroma and, sometimes DCIS cells. Therefore, RNA or protein in vitro extracts from whole tissue lysates also represent the presence of a variety of cell populations in the tissue under examination and potentially limits the ability to identify tumor cell-specific signals. As a consequence, several methods have been reported for tissue microdissection, including gross dissection of frozen tissue blocks [14], irradiation of manually ink-stained sections [15], touch preparations of frozen tissue specimens [16], macrodissection with manual tools [17-19], and laser capture microdissection [20]. The simplest of these methods is manual tool macrodissection of the FFPE slide. This has been the method adopted and is in routine use for the Oncotype Dx test, MammaTyper assay, and possibly other RNA-based tests. Recently, Laible et al. [21] showed that microdissection of FFPE tissue sections did not appear to be necessary for the MammaTyper assay, which assigns breast cancer specimens into the molecular subtypes according to the mRNA 
Fig. 6 Area-specific distribution of mRNA expression in IDCA and IDCA with DCIS cohort. Scatterplots depicting distribution of the percentage epithelial tumor area $(\mathbf{a}, \mathbf{b})$ and percentage DCIS area $(\mathbf{c}, \mathbf{d})$ versus mRNA quantification for $\operatorname{ERBB2}(\mathbf{a}, \mathbf{c})$ and $\operatorname{ESRI}(\mathbf{b}, \mathbf{d})$ in IDCA and IDCA with DCIS cohort
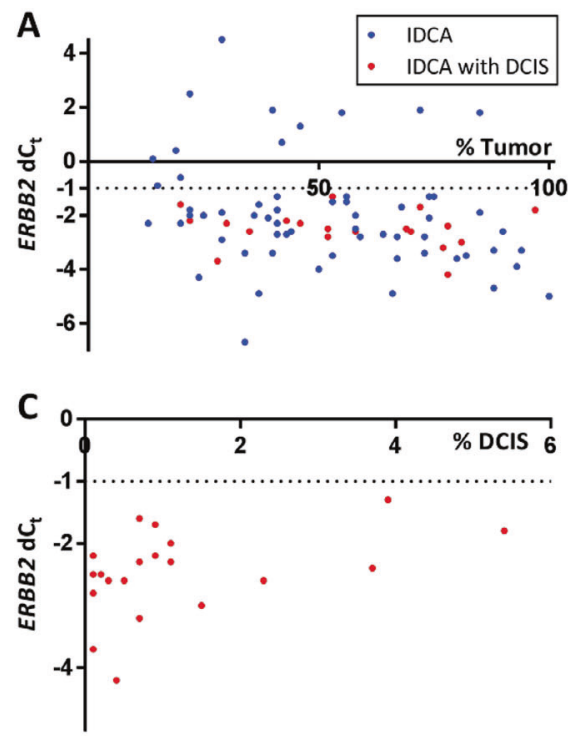

B
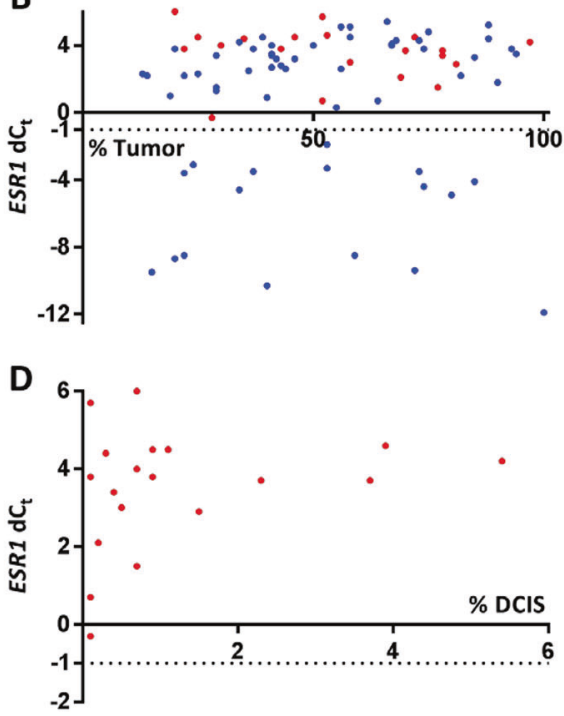

expression of ERBB2, ESR1, PGR, and MKi67. Our results with STRAT4 supports this study as we found no differences between results obtained using macrodissection and whole sections for the same targets. A few cases show discordance between RT-QPCR and IHC/FISH. This could be due to variance inherent in assays or actual discordance due to biological variance between RNA levels and protein levels, since not all RNA is translated. Similarly, not all cases with FISH amplification show increased protein and this may be a similar biological phenomenon.

IHC is the current practice for the measurements of HER2, ER, PR, and Ki-67 protein expression in breast cancer and ERBB2 status is assessed by FISH when HER2 IHC results are equivocal [10]. Over the years, the measurement of RNA for breast cancer markers has emerged using several commercially available platforms [22-24], most significantly for multiparameter prognostic testing. However, simple qRT-PCR has failed to gain acceptance for HER2 assessment. Here we showed a significant concordance $(P<0.001)$ between the GX closed system RTqPCR assay and IHC/FISH scoring for HER2, ER, and PR for the paired samples, non m-d versus $m-d$. These results suggest that the closed system RT-qPCR assay may have potential to be utilized in parallel to current practice for the measurements of HER2, ER, and PR.

There are a number of limitations to this work. Perhaps the most significant is that it is underpowered to assess concordance with IHC. While we show concordance here, the main focus of this work is the comparison with m-d vs non $\mathrm{m}$-d. Other larger studies are underway to assess concordance between IHC and RT-PCR. Since mixed population of invasive carcinoma and DCIS can create discrepancies in biomarker expression, future studies might address this issue by focusing on cases with HER2 overexpression in DCIS, but not in an adjacent invasive component. Also, since this work focused on HER2, we did not assess cases with small foci of ER/PR-negative invasive carcinoma that have significant amounts of benign glandular tissue. This could contribute to false positive results and may be addressed in future studies. Another limitation of this work is the focus on core needle biopsies. While our intention was only to assess small specimens (core biopsies), in the future we will attempt to assess FNA and whole, fullsized resection specimens. The conclusions of this work cannot necessarily be applied to other specimen types.

\section{Conclusions}

In summary, we compared mRNA expression for key breast cancer markers in non $\mathrm{m}-\mathrm{d}$ versus $\mathrm{m}-\mathrm{d}$ in IDCA and IDCA with DCIS cohorts and found that $\mathrm{m}$-d of whole sections is not required for accurate assessment of these genes in a closed system RT-qPCR assay. The simplicity of the assay workflow may be particularly valuable in low-resourced settings where routine access to pathology expertise and to high-quality IHC/FISH is challenging.

Acknowledgements We would like to thank Lori Charette and the team at the Yale Pathology Tissue Service for production of the highquality tissue sections used in this study. This work was supported by a research agreement from Cepheid.

\section{Compliance with ethical standards}

Conflict of interest D.L.R. is a consultant or advisor to Astra Zeneca, Agendia, Bethyl Labs, Biocept, BMS, Cell Signaling Technology, ClearSight, Merck, OptraScan, Perkin Elmer, and Ultivue. The remaining authors declare that they have no conflict of interest. 


\section{References}

1. Rizzardi AE, Johnson AT, Vogel RI, et al. Quantitative comparison of immunohistochemical staining measured by digital image analysis versus pathologist visual scoring. Diagn Pathol. 2012;7:42.

2. Choudhury KR, Yagle KJ, Swanson PE, et al. A robust automated measure of average antibody staining in immunohistochemistry images. J Histochem Cytochem. 2010;58:95-107.

3. Cregger M, Berger AJ, Rimm DL. Immunohistochemistry and quantitative analysis of protein expression. Arch Pathol Lab Med. 2006;130:1026-30.

4. Press MF, Slamon DJ, Flom KJ, et al. Evaluation of HER-2/neu gene amplification and overexpression: comparison of frequently used assay methods in a molecularly characterized cohort of breast cancer specimens. J Clin Oncol. 2002;20:3095-105.

5. Senkus E, Kyriakides S, Ohno S, et al. Primary breast cancer: ESMO Clinical Practice Guidelines for diagnosis, treatment and follow-up. Ann Oncol. 2015;26(Suppl 5):v8-30.

6. Fisher B, Redmond C, Brown A, et al. Influence of tumor estrogen and progesterone receptor levels on the response to tamoxifen and chemotherapy in primary breast cancer. J Clin Oncol. 1983;1:227-41.

7. Rose C, Thorpe SM, Lober J, et al. Therapeutic effect of tamoxifen related to estrogen receptor level. Recent Results Cancer Res. 1980;71:134-41.

8. Stierer M, Rosen H, Weber R, et al. Immunohistochemical and biochemical measurement of estrogen and progesterone receptors in primary breast cancer. Correlation of histopathology and prognostic factors. Ann Surg. 1993;218:13-21.

9. Sunderland MC, Osborne CK. Tamoxifen in premenopausal patients with metastatic breast cancer: a review. J Clin Oncol. 1991;9:1283-97.

10. Matos LL, Trufelli DC, de Matos MG, et al. Immunohistochemistry as an important tool in biomarkers detection and clinical practice. Biomark Insights. 2010;5:9-20.

11. Wolff AC, Hammond ME, Hicks DG, et al. Recommendations for human epidermal growth factor receptor 2 testing in breast cancer: American Society of Clinical Oncology/College of American Pathologists clinical practice guideline update. J Clin Oncol. 2013;31:3997-4013.

12. Wasserman BE, Carvajal-Hausdorf DE, Ho K, et al. High concordance of a closed-system, RT-qPCR breast cancer assay for HER2 mRNA, compared to clinically determined immunohistochemistry, fluorescence in situ hybridization, and quantitative immunofluorescence. Lab Invest. 2017;97:1521-1526.

13. Wu NC, Wong W, Ho K, et al. Xpert ${ }^{\circledR}$ Breast Cancer STRAT4* assay demonstrates high concordance with central IHC and FISH testing in FFPE breast tumor tissues. In: Poster presented at: 39th annual san antonio breast cancer symposium; December 6-10, 2016; San Antonio Breast Cancer Symposium: San Antonio, TX. http://sabcs16.posterview.com/nosl/p/P1-03-03.

14. Radford DM, Fair K, Thompson AM, et al. Allelic loss on a chromosome 17 in ductal carcinoma in situ of the breast. Cancer Res. 1993;53:2947-9.

15. Shibata D, Hawes D, Li ZH, et al. Specific genetic analysis of microscopic tissue after selective ultraviolet radiation fractionation and the polymerase chain reaction. Am J Pathol. 1992;141:539-43.

16. Kovach JS, McGovern RM, Cassady JD, et al. Direct sequencing from touch preparations of human carcinomas: analysis of p53 mutations in breast carcinomas. J Natl Cancer Inst. 1991;83:1004-9.

17. Emmert-Buck MR, Roth MJ, Zhuang Z, et al. Increased gelatinase A (MMP-2) and cathepsin B activity in invasive tumor regions of human colon cancer samples. Am J Pathol. 1994;145:1285-90.

18. Noguchi S, Motomura $\mathrm{K}$, Inaji $\mathrm{H}$, et al. Clonal analysis of predominantly intraductal carcinoma and precancerous lesions of the breast by means of polymerase chain reaction. Cancer Res. 1994;54:1849-53.

19. Zhuang Z, Vortmeyer AO. Applications of tissue microdissection in cancer genetics. Cell Vis. 1998;5:43-48.

20. Emmert-Buck MR, Bonner RF, Smith PD, et al. Laser capture microdissection. Science. 1996;274:998-1001.

21. Laible M, Schlombs K, Kaiser K, et al. Technical validation of an RT-qPCR in vitro diagnostic test system for the determination of breast cancer molecular subtypes by quantification of ERBB2, ESR1, PGR and MKI67 mRNA levels from formalin-fixed paraffin-embedded breast tumor specimens. BMC Cancer. 2016;16:398.

22. Park MM, Ebel JJ, Zhao W, et al. ER and PR Immunohistochemistry and HER2 FISH versus Oncotype DX: implications for breast cancer treatment. Breast J. 2014;20:37-45.

23. Duffy MJ, Walsh S, McDermott EW, et al. Chapter one-biomarkers in breast cancer: where are we and where are we going? In: Gregory SM, editor. Advances in clinical chemistry. Elsevier; 2015. p. 1-23.

24. Hagemann IS. Molecular testing in breast cancer: a guide to current practices. Arch Pathol Lab Med. 2016;140:815-24. 